\title{
Chronic care models and opportunities for improving health care practice and outcomes in Sub-Saharan Africa: Protocol for systematic review
}

Peter Otieno ( $\nabla$ pootienoh@gmail.com )

African Population and Health Research Center https://orcid.org/0000-0001-6828-8301

Charles Agyemang

Department of Public \& Occupational Health, Amsterdam UMC, University of Amsterdam

Razak M. Gyasi

African Population and Health Research Center(APHRC)

Anthony K. Ngugi

Aga Khan University

Catherine Kyobutungi

African Population and Health Research Center

Gershim Asiki

African Population and Health Research Center

\section{Protocol}

Keywords: chronic care models, non-communicable diseases, HIV/AIDS, comorbidities, multimorbidities, sub-Saharan Africa

Posted Date: August 17th, 2020

DOI: https://doi.org/10.21203/rs.3.rs-57249/v1

License: (c) (1) This work is licensed under a Creative Commons Attribution 4.0 International License.

Read Full License 


\section{Abstract}

Background: The rising burden of chronic diseases in sub-Saharan Africa (SSA) where health care systems are least developed has led to recent calls for increased investment in chronic care models (CCM) appropriate for low-resource settings. In SSA, limited resources are often allocated to treating acute diseases thus management of chronic conditions is a major challenge for health care systems. A large diversity of CCM exist in the literature but evidence supporting their use has been derived from high income settings. This is the motivation for conducting a systematic review to identify the existing CCM and their relevance and applicability in SSA.

Methods and analysis: All peer-reviewed published and grey literature on CCM will be included. PubMed/MEDLINE, Embase, Scopus, Web of Science and Global Health Cinahl, African Journals Online, Informit Online, and PsycINFO will be searched to identify relevant articles. OpenSIGLE will be reviewed for grey literature. The articles retrieved will be independently screened for inclusion by two independent investigators while a third reviewer will arbitrate the disagreements. An independent critical appraisal of retrieved studies will be performed using standardized critical appraisal checklists. The data will be extracted from the key elements of CCM. Outcome measures for the effectiveness of the models in the context of SSA will include any reported improvement to the health care service delivery or changes in the health outcomes of patients with chronic illnesses. The study findings will be reported according to the Preferred Reporting Items for Systematic Reviews (PRISMA).

Discussion: This systematic review is expected to generate crucial evidence on the applicability of CCM in SSA and unearth the CCM components that are suitable for different levels of health care delivery systems and the emerging needs of the people living with comorbidities and multimorbidities in SSA.

Systematic review registration: This review is registered in PROSPERO International Prospective Register of Systematic reviews CRD42020187756.

\section{Background}

Chronic diseases (CDs) are a major global public health challenge with non-communicable diseases (NCDs) accounting for $71 \%$ of the total global mortality while chronic infectious diseases such as HIV and TB account for over 2 million deaths annually [1, 2]. Before the HIV/AIDS epidemic, chronic diseases were invariably NCDs consisting of four main diseases including cardiovascular diseases, diabetes, cancer, and chronic respiratory diseases, which accounted for over $75 \%$ global NCD mortality [3]. There is an increasing prevalence of both communicable and non-communicable chronic diseases in low- and middle-income countries (LMICs) including sub-Saharan Africa (SSA) [2]. However, this increase is not matched with an appropriate health system response largely due to resource constraints in these settings. Most health services in SSA are still largely oriented to the provision of episodic health care and are generally not tailored to manage the projected demand for chronic care services. 
The chronic care model (CCM) first identified by McColl Institute for Healthcare Innovation at Group Health Cooperative [4], has been proposed as a solution to improve management, prevention of complications and outcomes in patients with chronic diseases [4]. This model identifies six essential elements including community resources and policies, health care organization, self-management support, delivery system design, decision support and clinical information systems. However, what constitutes a CCM and how it is implemented and delivered within healthcare services, has continued to evolve with the advent of new interventions. Crucially, CCM fosters productive interactions between informed patients who are actively involved in their care and experienced providers (nurses, physicians, pharmacists, etc.), resulting in a broadly applicable, higher quality and possibly more cost-effective patient care environment. Previous systematic review has shown that the implementation of CCM led to improved patient care and outcomes [5]. CCM has, therefore, been extensively elaborated and expanded upon by the World Health Organization to highlight macro issues related to population health and health promotion [6]. However, most chronic care models and systems have been developed largely in highincome countries with sophisticated health services [5], which may not appropriately apply in SSA. SSA has untapped capacity of chronic care models for HIV care which should be viewed as emerging models for other chronic diseases including NCDs [6]. Lessons learned from the implementation of care models for HIV and TB in SSA, could be effectively applied to improve care models for CDs [7].

A comprehensive evaluation of the applicability of CCM has not been systematically evaluated in SAA. We only came across one systematic review evaluating self-management as a component of CCM in SSA [8]. We, therefore, aim to conduct a systematic review to (1) describe the existing CCM globally; (2) determine which CCM components have been implemented in SSA with improved outcomes; (3) identify which combination of CCM components is suitable for different levels of health care delivery systems in SSA; and 4) identify the combination of CCM components that is suitable for the emerging needs of the people living with comorbidities and multimorbidities in SSA.

\section{Methods And Analysis Protocol registration}

This protocol has been registered in PROSPERO International Prospective Register for Systematic Reviews (CRD42020187756). The study findings will be reported according to the Preferred Reporting Items for Systematic Reviews (PRISMA) [9]. (See additional file 1).

\section{Eligibility criteria:}

1. The study must have any one or more of the following chronic diseases: cardiovascular diseases, diabetes, chronic respiratory diseases, cancers, and mental health, or HIV.

2. The study must have applied at least one of the six elements of CCM including community resources and policies, health care organization, self-management support, delivery system design, decision support and clinical information systems. 
3. The study must have been conducted between 1999 and 2020. We used 1999 because this was the beginning of the application of CCM.

4. The article is original research (e.g., clinical trials, experimental studies, etc.) or any type of review (e.g., narrative, systematic, meta-analysis, scoping, rapid, etc.).

\section{Exclusion criteria:}

1. The article is not focused primarily on CCM for NCDs and HIV

2. The article is about or uses animals as subjects.

\section{Information Sources}

Major electronic databases such as PubMed/MEDLINE, Embase, Scopus, Web of Science, Global Health Cinahl, Informit Online, African Journals Online, and PsycINFO will be searched for and identify relevant articles published in English. Citation and abstract databases will be conducted based on an agreed search strategy using combinations of keywords and controlled vocabulary when appropriate to address chronic disease management. Additional articles will be identified by scanning the reference list of all the relevant articles obtained through the search. Hand searching of key journals will also be conducted. Citations obtained from the hand searching of journals will be added to those retrieved via the databases. Additionally, we will search for grey literature from OpenSIGLE and other organizational websites such as Ministry of Health reports, surveys, white papers and policy documents.

\section{Search strategy}

We have developed a literature search strategy using the medical subject headings (MeSH), BOOLEAN operator and key text words such as ("chronic condition" OR "chronic disease" OR "Chronic Illnesses" or HIV/AIDS) and ("chronic care models" OR chronic disease management" which limit the search to the title or abstract with the terms (strategy OR intervention OR model)). The search strategy for The MEDLINE (OVID) will be adapted to match the syntax and subject headings for the other databases. We will document the search strategy for MEDLINE (OVID), Embase, Web of Science and African Journals Online database.

\section{Study Records}

\section{Data management}

A priori developed data extraction tool will be used for screening and selection of articles based on the inclusion and exclusion criteria. The tool will be piloted with at least two reviewers on randomly selected 20 articles to ensure clarity and foster an understanding of the process. Each article will be reviewed by 2 reviewers with a third reviewer available to serve as a tie-breaker when there is a disagreement in scoring. 
We will first upload the search results on Endnote 12 software and remove all the duplicates. Rayyan [10], a mobile and a web-based software program that supports collaboration among the reviewers screening and selecting articles for systematic will be used to place the remaining articles.

\section{Selection process}

The titles and abstracts of the articles retrieved from the literature search will be independently screened for inclusion by two investigators. We will obtain all the full texts for selected titles and/or abstracts including those with uncertainty. A third reviewer will be available to serve as a tie-breaker when there is a disagreement between the 2 reviewers scoring. The reasons for exclusion will be documented accordingly.

\section{Data collection process}

Predefined standardized data extraction will be used by two independent assessors to extract data form to extract the data. The disagreements between the two reviewers will be resolved by a third reviewer. The corresponding authors for articles under review will be contacted in case of missing information by sending a maximum of three email requests. The most recent literature will be considered for studies appearing in more than one published article and each survey will be treated as a separate study in case of surveys appearing in one article with multiple surveys done at different time points. We will separate the data for multinational surveys to depict the findings at the country level.

\section{Data items}

The data will be extracted based on general information such as type of publication, authors, year, country and region, study design, setting, respondents, sample size, mean or median age or age range, types of CCM studied including their respective elements such as community resources and policies, health care organization, self-management support, delivery system design, decision support and clinical information systems.

\section{Outcomes and prioritization}

In addition to describing the existing CCM and their key elements, outcome measures for effectiveness of the models in the context of SSA will include any reported improvement to the health care service delivery or changes in the health outcomes of patients with chronic illnesses.

\section{Risk of bias in individual studies}


Two independent reviewers will perform independent critical appraisal of retrieved articles using standardized critical appraisal checklists. We will use the Cochrane Handbook for Systematic Reviews of Interventions to assess the risk of bias for randomized and non-randomized control trials [11]. The bias of cross-sectional, cohort studies, case studies and case series will be assessed using the Joanna Briggs critical appraisal tools [12]. The results of the risk of bias assessments for each individual study and the overall risk of bias of the entire set of included studies will be reported in narrative form and in tables.

\section{Data Synthesis}

We have adapted the data synthesis approach developed by Weingarten et al [13]. The outcome measures for effectiveness of CCM in the context of SSA will include health care professional adherence to guidelines, patient outcomes and physiological measures of disease, adherence to treatment, health service use, quality of life, risk behavior, satisfaction, health and functional status. All the key outcome measures from the studies included in this review will be recorded under each of the above-listed categories as well as under the corresponding CCM and the overarching elements including community resources and policies, health care organization, self-management support, delivery system design, decision support and clinical information systems. Given that the current study involves a comprehensive approach for the inclusion of studies, there is a likelihood of heterogeneity in the recorded data, hence a decision to perform a meta-analysis to explore the effect-sizes will be undertaken depending on our findings. Summary tables on the effectiveness of the outcomes by the elements of the chronic care models will be generated. All analyses will be performed using Stata version 15.

\section{Discussion}

The results of this systematic review will be published in accordance with the PRISMA guidelines [9]. Summary tables will be used to display the data on the implementation of CCM and the overarching elements including community resources and policies, health care organization, self-management support, delivery system design, decision support and clinical information systems including the measures for effectiveness of CCM in the context of SSA such as health care professional adherence to guidelines, patient outcomes and physiological measures of disease, adherence to treatment, health service use, quality of life, risk behaviour, satisfaction, health and functional status. This systematic review is expected to generate crucial evidence on the applicability of the CCM in SSA and unearth the CCM components that are suitable for different levels of health care delivery systems and the emerging needs of the people living with comorbidities and multimorbidities in SSA.

\section{Abbreviations}

CCM: chronic Care Models; HIV/AIDS: Human immunodeficiency virus/Acquired immune deficiency syndrome; LMICs: low and middle-income countries; MeSH: Medical Subject Headings; NCDs; Non- 
communicable diseases; PRISMA: Preferred Reporting Items for Systematic Reviews; PROSPERO:

Prospective Register of Systematic Reviews, SSA; Sub-Sahara Africa.

\section{Declarations}

\section{Ethics approval and consent to participate}

This study will not require ethics consideration as it based on published studies.

\section{Consent for publication}

Not applicable

\section{Availability of data and materials}

Not applicable

\section{Competing interests}

The authors declare that they have no competing interests.

\section{Funding}

This study is funded by the Stichting, Joep Lange Institute for Global Health and Development and Dutch Ministry of Foreign Affairs (BuZa) under the Joep Lange Chair grant for Catherine Kyobutungi. The views expressed here are those of the author(s) and not necessarily those of the Joep Lange Institute for Global Health and Development.

\section{Authors' contributions}

POO conceived and designed the initial study. All authors contributed to the conceptualization and design of the study. POO drafted the initial manuscript. All authors contributed to the development of the selection criteria, the risk of bias assessment strategy, and data extraction criteria. POO is the guarantor of the review. All authors read, provided feedback, and approved the final manuscript prior to submission.

\section{Acknowledgements}

Not applicable 


\section{References}

1. World Health Organization, Noncommunicable diseases: Key facts. 2018, World Health Organization website.

2. Roth GA, et al. Global, regional, and national age-sex-specific mortality for 282 causes of death in 195 countries and territories, 1980-2017: a systematic analysis for the Global Burden of Disease Study 2017. The Lancet. 2018;392(10159):1736-88.

3. Abajobir AA, et al., Global, regional, and national age-sex specific mortality for 264 causes of death, 1980-2016: a systematic analysis for the Global Burden of Disease Study 2016. 2017.

4. Wagner EH, Austin BT, Von MK. Improving outcomes in chronic illness. Managed care quarterly. 1996;4(2):12-25.

5. Battersby MW. Health reform through coordinated care: SA HealthPlus. Bmj. 2005;330(7492):662-5.

6. Geldsetzer P, Ortblad K, Bärnighausen T. The efficiency of chronic disease care in sub-Saharan Africa. BMC Med. 2016;14(1):127.

7. Harries AD, et al., Adapting the DOTS framework for tuberculosis control to the management of noncommunicable diseases in sub-Saharan Africa. PLoS medicine, 2008. 5(6).

8. Aantjes CJ, Ramerman L, Bunders JF. A systematic review of the literature on self-management interventions and discussion of their potential relevance for people living with HIV in sub-Saharan Africa. Patient Educ Couns. 2014;95(2):185-200.

9. Shamseer $\mathrm{L}$, et al., Preferred reporting items for systematic review and meta-analysis protocols (PRISMA-P) 2015: elaboration and explanation. Bmj, 2015. 349.

10. Ouzzani M, et al. Rayyan-a web and mobile app for systematic reviews. Systematic reviews. 2016;5(1):210.

11. Green S, et al. Cochrane handbook for systematic reviews of interventions: Cochrane book series. Naunyn-Schmiedeberg's Arch Exp Pathol Pharmakol. 2008;5:S38.

12. Joanna Briggs Institute. Joanna Briggs Institute reviewers' manual: 2014 edition, in Australia: The Joanna Briggs Institute. 2014.

13. Weingarten SR, et al. Interventions used in disease management programmes for patients with chronic illnesswhich ones work? Meta-analysis of published reports. Bmj. 2002;325(7370):925.

\section{Supplementary Files}

This is a list of supplementary files associated with this preprint. Click to download.

- additionalfile1PRISMAchecklist.docx 